\title{
A NOTE ON FINITE METABELIAN $p$-GROUPS ${ }^{1}$
}

\section{J. D. GILLAM}

Abstracr. Let $A$ be an abelian subgroup of maximal order in the finite metabelian $p$-group $P$. It is shown that there exists a normal abelian subgroup $A_{1}$ of $P$ such that the order of $A_{1}$ is equal to the order of $A$.

In [1], J. L. Alperin raised the following question. If $p$ is any prime and $A$ is an abelian subgroup of index $p^{n}$ in the finite $p$-group $P$, does there exist a normal abelian subgroup of $P$ of index $p^{n}$ ? Alperin has shown in [1] that the answer to this question is yes, if $n$ is 2 or 3 . The purpose of this note is to answer this question in the affirmative in the special case that $P$ is metabelian. As in [2], for any $p$-group $P$ we let $A(P)$ be the set of abelian subgroups of $P$ of maximal order. We shall prove that if $P$ is metabelian there exists a normal subgroup $A$ belonging to $A(P)$. For metabelian p-groups, this clearly implies an affirmative answer to Alperin's question. The notations and terminology are standard.

Lemma 1. Suppose $P$ is a finite metabelian p-group and $A$ belongs to $A(P)$. Then the following are true.

(i) If $x \in P,[x, A] C_{A}[x, A] \in A(P)$.

(ii) $[x, y, z][z, x, y][y, z, x]=1$ for all $x, y, z$ in $P$.

(iii) If $x \in P$, the order of $A / C_{A}[x, A]$ is equal to the order of $[x, A] / A \cap[x, A]$.

(iv) If $x \in P, C_{A}[x, A]=A \cap A^{x}$ and $C_{A}[\langle x\rangle, A]=\bigcap\left(A^{y}: y \in\langle x\rangle\right)$.

Proof. Statement (i) is contained in [2, Theorem 2.4, p. 272]. Statement (ii) is well known and follows directly from [2, Theorem 2.3, p. 19]. The third statement follows from (i), since the order of $A$ is equal to the order of $[x, A] C_{A}[x, A]$. For (iv), $a \in C_{A}[x, A]$ if and only if $a \in A \cap C_{A}\left(\left(b^{-1}\right)^{x}\right)$ for all $b \in A$, if and only if $a \in A \cap C_{P}\left(A^{x}\right)$ $=A \cap A^{x}$. The second part of (iv) follows in exactly the same way.

Lemma 2. Suppose $P$ is a finite metabelian p-group, $A \in A(P)$, and $x \in P$ is such that $[x, A] \leqq N_{P}(A)$. Then $C_{A}[x, A]=C_{A}[\langle x\rangle, A]$, and so $[\langle x\rangle, A] C_{A}[\langle x\rangle, A] \in A(P)$.

Received by the editors September 25, 1969.

A MS Subject Classifications. Primary 2025, 2040.

Key Words and Phrases. Metabelian p-group, abelian subgroup of maximal order.

1 After submitting this note, the author became aware that J. L. Alperin has proved the result, as well as some stronger results. 
Proof. Define the map $f: A \rightarrow[x, A] / A \cap[x, A]$ by $f(a) \equiv[x, a]$ $(\bmod A \cap[x, A])$ for all $a \in A$. If $a_{1}, a_{2} \in A$, then $\left[x, a_{1} a_{2}\right]=\left[x, a_{2}\right]$ $\cdot\left[x, a_{1}\right]\left[x, a_{1}, a_{2}\right]$ and $\left[x, a_{1}, a_{2}\right] \in A \cap[x, A]$ by hypothesis. Since $P$ is metabelian, $f$ is a homorphism, and $f$ is clearly an onto map. If $a \in \operatorname{Ker}(f)$, then $[x, a] \in A$, and so $\left(a^{-1}\right)^{x} \in A$. Hence $a \in A \cap A^{x^{-1}}$. Also since $[x, a] \in A$ we have $\left[x, a, a_{1}\right]=1$ for all $a_{1}$ in $A$. By $[2$, Lemma 2.5 , p. 20] we obtain $\left[x, a_{1}, a\right]=1$ for all $a_{1}$ in $A$, and therefore $a \in C_{A}[x, A]=A \cap A^{x}$. Therefore $\operatorname{Ker}(f) \leqq A \cap A^{x} \cap A^{x^{-1}} \leqq A \cap A^{x}$, but by Lemma 1 we must have equalities. It now follows easily that $C_{A}[x, A]=A \cap A^{x}=\bigcap\left(A^{y}: y \in\langle x\rangle\right)=C_{A}[\langle x\rangle, A]$. Since $[\langle x\rangle, A]$ $\geqq[x, A],[\langle x\rangle, A] C_{A}[\langle x\rangle, A] \in A(P)$.

Theorem. If $P$ is a finite metabelian p-group, then there exists $A \in A(P)$ such that $A$ is normal in $P$.

Proof. Let $A \in A(P)$ and $M$ be a maximal subgroup of $P$ containing $A$. Inductively we may assume $A$ is normal in $M$. Choose $x \in P$ such that $P=\langle x, M\rangle$. Then $[x, A] \leqq M \leqq N_{P}(A)$, and by Lemma $2, A_{1}=[\langle x\rangle, A] C_{A}[\langle x\rangle, A] \in A(P)$. We now show that $A_{1}$ is normal in $P$. Trivially $x \in N_{P}[\langle x\rangle, A]$. Let $m \in M$ and $\left[x^{i}, a\right]$ be a generator of $[\langle x\rangle, A]$. Then $\left[x^{i}, a\right]^{m}=\left[x^{i}, a\right]\left[x^{i}, a, m\right]$ and by Lemma 1 , $\left[x^{i}, a, m\right]=\left[a, m, x^{i}\right]^{-1}\left[m, x^{i}, a\right]^{-1}$ which belongs to $A_{1}$. Let $a_{1}$ $\in C_{A}[\langle x\rangle, A]$, then $a_{1}^{x}=a_{1}\left[a_{1}, x\right] \in A_{1} ;$ if $m \in M$, then $\left[a_{1}^{m},[\langle x\rangle, A]\right]$ $=\left[a_{1},[\langle x\rangle, A]^{m^{-1}}\right]^{m} \leqq\left[a_{1}, A_{1}\right]^{n}=\langle 1\rangle$. Therefore $P=\langle x, M\rangle$ normalizes $A_{1}$.

\section{REFERENCES}

1. J. L. Alperin, Large Abelian subgroups of p-groups, Trans. Amer. Math. Soc. 117 (1965), 10-20. MR $30 \# 1180$.

2. D. Gorenstein, Finite groups, Harper \& Row, New York, 1968. MR 38 \#229.

Michigan State University, East Lansing, Michigan 48823 and

Central Michigan University, Mt. Pleasant, Michigan 48858 